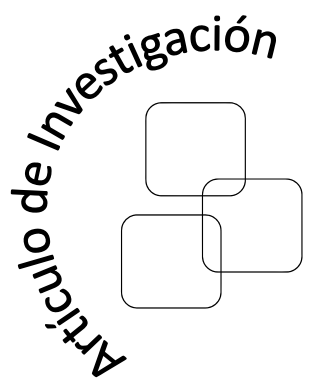

Carlos Franco Franco Universidad Católica de Colombia cafranco@ucatolica.edu.co

Germán Andrés Méndez Giraldo Universidad Distrital Francisco José de Caldas gmendez@udistrital.edu.co

John Espitia

Universidad Distrital Francisco José de Caldas jaerjr@gmail.com

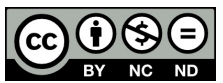

Citación: Franco, C., Méndez, G. A. y Espitia, J.(2015). Evaluación de escenarios de descongestión vehicular en Bogotá D.C. mediante Dinámica de Sistemas.

En: Ingeniería, Vol. 20, No. 2, pp. 209-231

(C)Los autores; titular de derechos de reproducción Universidad Distrital Francisco José de Caldas

En línea DOI: http://dx.doi.org/10.14483/udistrital.jour.reving.2015.1.a03

\section{Evaluación de escenarios de descongestión vehicular en Bogotá D.C. mediante Dinámica de Sistemas}

\section{Assessment of scenarios of vehicular decongestion in Bogotá D.C., using system dynamics}

\section{Resumen}

Este trabajo propone el desarrollo de un modelo basado en Dinámica de Sistemas en el cual se caracteriza el sistema de transporte de Bogotá D.C. Para realizar lo anterior se modelan las principales variables que intervienen en el sistema de transporte, a fin de lograr un análisis global de la problemática de transporte y evaluar posibles soluciones propuestas por diferentes expertos.

El modelo es desarrollado mediante el software Stella, donde se incluyen los diferentes actores que intervienen en la problemática global. Previo al desarrollo del modelo computacional, se lleva a cabo la recolección de información a través de expertos en el tema, que permite realizar una caracterización inicial, para poder así comprender la relación entre variables. Adicionalmente se evalúan sobre el modelo seis diferentes alternativas de solución a la problemática, con el propósito de observar el impacto que estas tendrían sobre el sistema de transporte.

Palabras claves: Dinámica de Sistemas, pensamiento sistémico, transporte urbano

\section{Abstract}

This paper proposes the development of a model based on system dynamics in which the transportation system in Bogotá D.C. is characterized. To do this the main variables involved in the transport system are modeled in order to achieve a global analysis of the problems of transportation and evaluate possible solutions proposed by different experts.

The model is developed using the software Stella, and the different actors involved in global issues are included. Prior to the development of computational model is carried out data collection by experts on the subject, which shall provide an initial characterization, in order to understand the relationship between variables. Additionally six different alternative solutions to the problem are evaluated, to observe the impact that they would have on the transportation system.

Key words: systems dynamics, systems thinking, urban transportation 


\section{Introducción}

Las temáticas de movilidad urbana y transporte colectivo son de vital importancia para las ciudades, ya que cuando se logra un progreso apropiado en estos temas se alcanza un buen desarrollo colectivo de la ciudad; es tal la relevancia que tiene la movilidad, que se reconoce como la primera necesidad colectiva de cualquier tipo de ciudad [1]. Para el Distrito Capital la movilidad urbana y el transporte colectivo no son una problemática ajena, puesto que diariamente la ciudad se ve inmersa en los problemas que trae el no tener una buena planificación en estas áreas, ya que se presenta una ineficiencia de los diferentes actores involucrados en el sistema de transporte de la ciudad.

Con el propósito de afrontar esta problemática, las diferentes administraciones han implementado medidas para tratar de mitigar los efectos adversos que traen los problemas de movilidad en la ciudad. La primera solución que se planteó para este problema fue la implementación de sistema Transmilenio, que ha contribuido a solucionar en parte la problemática de movilidad en las zonas donde presta su servicio [2]. La siguiente solución que se implementó es la del pico y placa, con lo cual se restringe la circulación de una cantidad de vehículos en ciertas franjas horarias, con el fin disminuir el uso del espacio por vehículos privados y de servicio público; esta medida fue adoptada desde el año 1998 con la administración del alcalde Enrique Peñalosa. Finalmente, aunque no está implementado en su totalidad, la última estrategia que se adoptó consiste en utilizar un sistema integrado de transporte público que conecte los diferentes actores del transporte colectivo, dicha estrategia surge como decreto desde el año 2009 y hoy en día se encuentra en la fase de implementación.

En el presente artículo se aborda la problemática a través de Dinámica de Sistemas, pues, como lo mencionó Jay Forrester en su libro [3], los sistemas sociales, que son de gran complejidad, se pueden abordar mediante modelos computacionales para lograr un mejor entendimiento, ya que la manera que se tiene para abordar este tipo de problemáticas es mediante modelos o patrones de pensamiento y estos pueden tener perjuicios o alteraciones.

A fin de hacer frente al problema de movilidad del Distrito capital se siguieron los lineamientos de la metodología propuesta por los docentes Lindsay Álvarez y Germán Méndez [4], que consiste en la consulta bibliográfica de la situación problémica de autores tanto nacionales como internacionales, después se construye un diagrama de afinidad en donde se agrupen las características consideradas por los expertos para la problemática; el siguiente paso consiste en la elaboración de un diagrama de Pareto en donde se muestran las características provenientes del diagrama de afinidad, y posteriormente diseñar un diagrama causa-efecto que contemple las variables principales que intervienen en la problemática de transporte. Siguiendo con la metodología se diseña un diagrama causal en donde se muestran las diferentes variables que intervienen en la situación problema y, además, se muestra la interacción entre ellas, a continuación se consultan diferentes fuentes de datos para así determinar modelos de regresión que representen el sistema, por último se usa un software diagramático que para este caso es STELLA, el cual permite mostrar las interrelaciones entre variables con su respectivo modelo matemático.

Después de desarrollar el modelo computacional se evalúan seis alternativas propuestas por diferentes autores para solucionar la problemática de movilidad. Sobre cada uno de estos escenarios se observará el impacto que tendrían sobre indicadores de movilidad y se hará énfasis 
en el indicador de velocidad promedio, el cual es uno de los indicadores más utilizados para evaluar un sistema de movilidad.

\section{Estudios relacionados}

De otro lado, se han realizado diversos estudios relacionados con la movilidad en distintas partes del mundo utilizando Dinámica de Sistemas, inclusive podemos encontrar varios estudios dentro de Colombia que se han presentado para estudiar la problemática local. A continuación se enunciarán algunos de estos, tanto internacionales como locales, para así poder encontrar las diferencias con el trabajo propuesto en el presente artículo.

Dentro de los primeros trabajos que se han realizado se pueden encontrar diferentes modelaciones para abordar la problemática, uno de ellos en Delhi [5]; en este trabajo se elaboran experimentos para medir los efectos en la congestión vial, la distribución del modo de transporte, el consumo de transporte y la contaminación generada por el tráfico. Para hacer esta modelación los autores tienen en cuenta cuatro sectores principales: socio-económico, transporte de pasajeros, medio ambiente y energía. Las políticas que se evalúan sobre el modelo de simulación hacen referencia al costo del combustible, el incremento de los impuestos sobre vehículos particulares y la disminución de los costos de viaje en trenes. El propósito del artículo es evaluar dichas políticas a fin de reconocer si efectivamente disminuyen la congestión y la contaminación ambiental.

Otra de las primeras aproximaciones al modelamiento de transporte mediante Dinámica de Sistemas fue la modelación de sistemas de transporte con respecto a nuevas ciudades en Egipto [6]. Al final del artículo se hace una descripción rigurosa de cómo la Dinámica de Sistemas contribuye a la modelación de los sistemas de transporte.

Estudios recientes que se han presentado incluyen nuevas variables. Es así que en [7], se presenta una modelación del sistema de transporte en Dalian China, en donde se modelan siete submodelos que interactúan entre sí para representar el sistema global, estos submodelos son: desarrollo económico, población, número de vehículos, influencia ambiental, demanda de viajes, congestión de tráfico y planeación de transporte. La conclusión a la cual llegan con el modelo es que se debe restringir el número de vehículos con el fin de lograr una sostenibilidad y eficiencia en el sistema de transporte.

Finalmente, existen otras aproximaciones basadas en Dinámica de Sistemas, que no estudian casos particulares de ciudades, sino que realizan análisis de políticas de sostenibilidad de transporte [8], aproximaciones a viajes entre ciudades [9], y evaluaciones del impacto de trenes sobre regiones metropolitanas [10].

No solo existen aproximaciones en ciudades fuera de Colombia, también hay trabajos que hacen aproximaciones a la problemática con Dinámica de Sistemas dentro de nuestra ciudad, entre estos trabajos se encuentran [11] [12] y [13]. En los tres trabajos hay una alta concentración en la modelación de los sistemas de transporte en ciudades de Colombia, en este caso Bogotá D.C. y Cali, para saber cuál es el impacto sobre el transporte urbano. 
Con estos análisis de trabajos desarrollados en la temática se muestra la novedad del trabajo presentado en este artículo, ya que en él se desarrolla una modelación global de todos los componentes que hacen parte del sistema de movilidad en la ciudad de Bogotá D.C., además de utilizar una metodología nueva para el análisis de sistemas.

\section{Conceptos previos de Dinámica de Sistemas}

A partir del año 1950 se desarrolló una metodología para estudiar comportamientos de sistemas complejos en donde se buscaba principalmente elaborar políticas para sistemas complejos mediante el uso de herramientas computacionales, estos sistemas compartían una característica esencial: su comportamiento estaba regido por un componente básico denominado los bucles de retroalimentación.

Los elementos principales de la Dinámica de Sistemas son los siguientes [20]:

- Diagramas causales

- Ciclos de realimentación

- Retrasos

- Estructuras genéricas

El diagrama causal es aquel que muestra la relación entre cada par de variables y da su sentido de relación. Para obtener un diagrama causal se realiza para cada par de variables una flecha en la cual se utiliza un signo para indicar si la relación es positiva o negativa, figura 1.

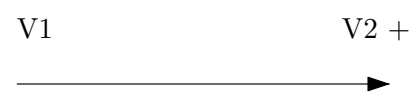

Figura 1. Ejemplo diagrama causal

En la figura 1 se muestra la relación de las variables 1(V1.) y 2(V2.), y el signo positivo de la relación, lo que indica que a cualquier aumento de la variable 1, existe un crecimiento de la variable 2 (no tiene que ser en la misma proporción), y ocurre lo mismo en el caso de que la variable 1 decrezca. Una relación negativa indica que a un aumento de la variable 1 generará un decrecimiento de la variable 2.

Los ciclos de realimentación son aquellos que muestran la información del estado del sistema en un momento dado, cuando este sistema ha sido sometido a una alteración. Los ciclos pueden ser positivos o negativos, un ciclo positivo indica que todas las conexiones entre variables son positivas, lo que quiere decir que siempre hay un refuerzo positivo en el comportamiento; por otro lado, un bucle negativo representa las situaciones en las cuales se quiere alcanzar una meta o lograr un objetivo.

Cuando existen unas relaciones entre dos variables, la influencia de una variable sobre otra puede no ser en el mismo momento de tiempo, es decir que la influencia de esta variable tarda 
en manifestarse, esto es lo que se denomina un retraso o una demora. Estos retrasos pueden determinar las posibles decisiones que se tomen sobre un sistema. Finalmente las estructuras genéricas hacen referencia a aquellos comportamientos en los cuales existen sistemas de realimentación positiva y negativa a la vez, que guardan ciertos comportamientos que pueden caracterizar situaciones cotidianas.

El último componente hace referencia al traspaso del modelo causal al modelo computacional, en este paso se crean los diagramas de Forrester [20]. Los componentes del diagrama de Forrester son los siguientes:

- Niveles: son aquellos que acumulan los valores de las variables de interés y muestran la situación o estado del sistema en cada instante del modelo. Los elementos que hacen que aumenten o disminuyen los valores de los niveles son los flujos.

- Flujos: son aquellos que modifican los niveles y toman los valores respectivos de las acciones o decisiones que son implementadas en el modelo.

- Variables auxiliares: son parámetros que sirven como elementos de entrada para los flujos.

\section{Desarrollo de la metodología}

A continuación se muestran la metodología utilizada, junto con los resultados y el análisis de cada uno de los pasos. Dentro de los pasos utilizados se encuentran los siguientes:

- Consulta de expertos

- Diagrama de Pareto

- Diagrama causa-efecto

- Modelo de simulación

- Análisis de políticas

\subsection{Consulta de expertos}

En total se consultaron 50 expertos entre nacionales e internacionales, de los cuales se tomó la problemática planteada para la movilidad en Bogotá D.C, o para otra ciudad en particular, de estos autores se obtuvieron 350 causas, el resumen de los autores consultados se muestra en la tabla I; las principales características a la problemática señalada por los expertos se muestran en la tabla II, estas se agruparon en 14 causas mediante afinidad, en donde se agrupan aquellas que tienen objetivos o enunciados similares. 
Tabla I. Características de los autores consultados

\begin{tabular}{lcc}
\hline Característica & Valor & Porcentaje \\
\hline Autor (es) colombianos & 31 & $62 \%$ \\
Autor (es) extranjeros & 19 & $38 \%$ \\
Problemática de Bogotá & 35 & $70 \%$ \\
Problemática internacional & 15 & $30 \%$ \\
Fuentes de hasta cinco años & 31 & $62 \%$ \\
Fuentes de más de cinco años & 19 & $38 \%$ \\
\hline
\end{tabular}

Como se observa en la tabla I, la mayor parte de expertos consultados son de nacionalidad colombiana, pues representan un $62 \%$ del total de autores consultados, además la mayoría de ellos hace referencia en sus artículos a la problemática local, teniendo un $70 \%$ de autores que hablan sobre la movilidad en Bogotá; asimismo la mayor parte de ellos son fuentes recientes, pues el $62 \%$ no superan los cinco años.

Tabla II. Características señaladas por los autores

\begin{tabular}{lcccc}
\hline Causa de la problemática & Frecuencia & Porcentaje & $\begin{array}{c}\text { Frecuencia } \\
\text { acumulada }\end{array}$ & $\begin{array}{c}\text { Porcentaje } \\
\text { Acumulado }\end{array}$ \\
\hline Gestión deficiente en la planeación de la movilidad & 51 & $14,6 \%$ & 51 & $14,6 \%$ \\
Uso indiscriminado del automóvil & 38 & $10,9 \%$ & 89 & $25,4 \%$ \\
Modelo empresarial en el sector transporte & 36 & $10,3 \%$ & 125 & $35,7 \%$ \\
Infraestructura vial & 33 & $9,4 \%$ & 158 & $45,1 \%$ \\
Incremento del parque automotor & 27 & $7,7 \%$ & 185 & $52,9 \%$ \\
Ordenamiento territorial & 27 & $7,7 \%$ & 212 & $60,6 \%$ \\
Calidad del transporte público y masivo & 25 & $7,1 \%$ & 237 & $67,7 \%$ \\
Debilidades institucionales & 23 & $6,6 \%$ & 260 & $74,3 \%$ \\
Sobreoferta de transporte & 22 & $6,3 \%$ & 282 & $80,6 \%$ \\
Desintegración de sistemas de transporte público & 18 & $5,1 \%$ & 300 & $85,7 \%$ \\
Congestión vial & 16 & $4,6 \%$ & 316 & $90,3 \%$ \\
Tasa de accidentalidad & 12 & $3,4 \%$ & 328 & $93,7 \%$ \\
Cultura ciudadana & 11 & $3,1 \%$ & 339 & $96,9 \%$ \\
Ilegalidad & 11 & $3,1 \%$ & 350 & $100 \%$ \\
\hline
\end{tabular}

En la tabla II se muestra al detalle cuáles son las causas señaladas por los expertos consultados, existe una causa de la problemática que se denomina Administración Distrital, que según la consulta realizada se puede representar mediante tres causas diferentes: ordenamiento territorial, debilidades institucionales y gestión deficiente de planeación de la movilidad. Además de las causas señaladas se muestra la frecuencia de cada una de ellas, el porcentaje, la frecuencia acumulada y el porcentaje acumulado, esto con el fin de tener un insumo de entrada para el siguiente paso de la metodología y lograr obtener el diagrama de Pareto.

\subsection{Diagrama de Pareto}

Utilizando las características mostradas en la tabla II y el porcentaje acumulado se elabora el diagrama de Pareto [14], que se muestra en la figura 2; para elaborarlo se asigna un valor numérico a cada característica, tabla III. 
Tabla III. Identificación de las características

\begin{tabular}{ll}
\hline Identificación & \multicolumn{1}{c}{ Causa de la problemática } \\
\hline 3,3 & Gestión deficiente en la planeacion de la movilidad \\
2 & Uso indiscriminado del automóvil \\
4 & Modelo empresarial en el sector transporte \\
11 & Infraestructura vial \\
1 & Incremento del parque automotor \\
3,1 & Ordenamiento territorial \\
8 & Calidad del transporte público y masivo \\
3,2 & Debilidades institucionales \\
5 & Sobreoferta de transporte \\
9 & Desintegración de sistemas de transporte público \\
12 & Congestión vial \\
6 & Tasa de accidentalidad \\
7 & Cultura ciudadana \\
10 & Ilegalidad \\
\hline
\end{tabular}

La enumeración mostrada en la tabla III va de unidad en unidad, pero las características 3,1, 3,2 y 3,3 como hacen parte de Administración Distrital, tienen una enumeración diferente a las demás, esto para enmarcarlos dentro del mismo conjunto.

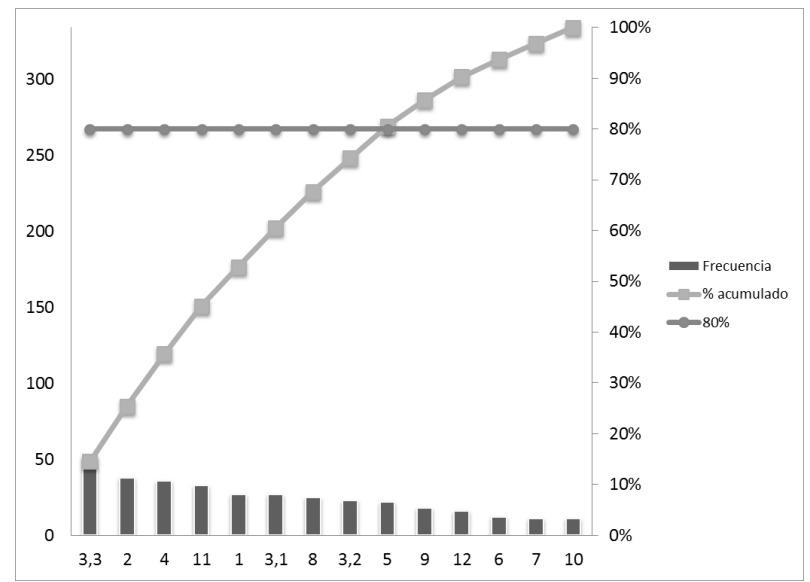

Figura 2. Diagrama de Pareto.

De los resultados ilustrados en la figura 2 se infiere que no se cumple la proporción del 80-20, lo que se traduce en que todas las problemáticas encontradas tienen igual importancia y por tanto no deben ser tratadas por separado, de allí se deduce la primera conclusión parcial y distinción con los trabajos realizados sobre la problemática de transporte en Bogotá D.C., ya que en dichos trabajos se encuentran priorizadas algunas características del sistema, pero nunca se han tenido en cuenta en su totalidad para obtener un análisis global de esta. 


\subsection{Diagrama causa-efecto}

El desarrollo del diagrama causa efecto y el diagrama causal se basó en las características mostradas en la tabla 2. Se realizaron dos diagramas, uno general y uno específico que se muestran en las figuras 2 y 3 respectivamente; en estos diagramas se evidencian las relaciones existentes entre las diferentes variables consideradas en la investigación, en su construcción se tomó como base el pensamiento sistémico para evaluar las interacciones entre las variables [15].

Las variables que se identificaron para el diagrama causa-efecto son las siguientes:

- Planeación de movilidad: son todos aquellos modelos de planeación de movilidad que son usados por el Distrito para integrar el sistema de transporte en el sistema territorial, con el propósito de garantizar la accesibilidad física y financiera a todos los actores de la ciudad.

- Semaforización y señalización: hace referencia al aumento de semáforos y señales viales en la ciudad.

- Construcciones viales: es todo crecimiento de la red vial del sistema de transporte de Bogotá D.C.

- Mantenimiento: incluye los arreglos preventivos y correctivos a la maya vial de la ciudad.

- Infraestructura vial: la malla vial de Bogotá D.C. se encuentra constituida por dos subsistemas principales, el subsistema vial, conformado por la malla vial arterial, intermedia y local, y el subsistema de transporte que lo componen las troncales y Transmilenio. A fin de hacer referencia a las condiciones de las vías se usa un índice de condición de pavimento, el cual permite calificar la condición superficial de su estructura; este índice es conocido como el IPC, el cual clasifica el estado de la malla vial en vías en mal estado, regular estado y buen estado.

- Ilegalidad: hace referencia a la prestación del servicio de transporte, tanto colectivo como individual, por medios de transporte no autorizados por el Distrito.

- Parque automotor: hace referencia a la cantidad del parque automotor en la ciudad de Bogotá D.C.

- Congestión: está enlazado con el espacio ocupado por la cantidad de vehículos circundantes en la ciudad.

- Velocidad promedio: indica la media de velocidad con la que se transportan los bogotanos en la ciudad.

- Cultura ciudadana: existe una cultura ciudadana relacionada con aquellos comportamientos, tanto de conductores como de peatones que generan un espacio en donde se mantiene la eficiencia del transporte y la seguridad vial.

- Accidentalidad: se refiera a la cantidad de accidentes viales en la ciudad. 
- Tiempo entre viajes: está relacionada con la velocidad promedio e indica el tiempo promedio que toma en realizar un desplazamiento en la ciudad.

- Tasa de ocupación vial: es la cantidad de vehículos que ocupan un área determinada de vías.

- Edad parque automotor: hace referencia a cuál es la edad promedio del parque automotor que influye directamente en la contaminación y en la renovación de la flota vehicular.

- Contaminación: es la cantidad de contaminación que aportan los carros y transporte público al ambiente.

- Calidad del transporte: hace referencia a los niveles de servicio en la prestación del servicio de transporte público y colectivo.

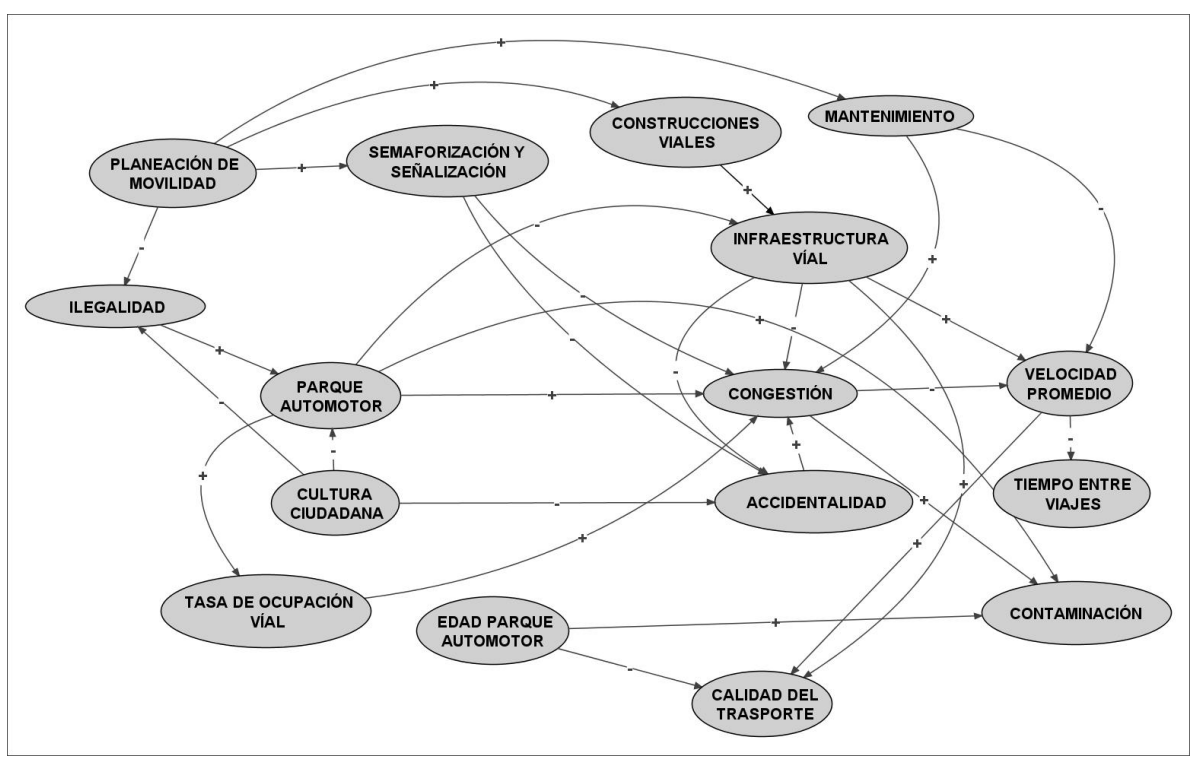

Figura 3. Diagrama general.

Como se puede observar en la figura 3, existen diferentes interacciones; así, podemos definir que el comportamiento de la planeación de movilidad influye directamente sobre cuatro variables: ilegalidad, semaforización y señalización, construcciones viales y por último el mantenimiento. El aumento de planeación de movilidad ocasiona un aumento en la semaforización y señalización ya que se estudian y analizan las intersecciones de la ciudad en donde se requieren estos elementos.

A su vez la semaforización está relacionada con dos variables, congestión y accidentalidad, a un aumento de la semaforización y señalización se obtiene una disminución de la congestión en tanto que la función de estos es solucionar problemas de espera en la malla vial y de flujo vehicular dentro de la misma, un aumento en la congestión genera un aumento en la contaminación a causa de que las emisiones de monóxido de carbono se producen en mayor nivel antes de los $30 \mathrm{~km} / \mathrm{h}$ [16]; por su parte, un aumento en la congestión conduce a una disminución de la velocidad promedio provocada por la condición de saturación que sufren 
los automóviles por un exceso de demanda de vías, lo que lleva finalmente a que por aumentos de velocidad promedio se generen reducciones en los tiempos de viaje, ya que representan el tiempo que tarda un sujeto entre su origen y destino, y al tener aumentos de la velocidad promedio estos tiempos disminuyen.

Por otra parte, un aumento en la señalización y semaforización contribuye a una disminución en las tasas de accidentalidad, ya que como se mencionó anteriormente la semaforización se encarga de regular los flujos de vehículos, al ser estos flujos controlados se reduce la probabilidad de accidente. Finalmente, al presentarse un aumento de las tasas de accidentalidad producen un aumento en la congestión a causa de que los accidentes en las vías de tránsito conducen a una utilización de espacio, por lo que se reduce la capacidad de la vía [17].

El aumento en la planeación de movilidad crea un aumento en las construcciones de malla vial y un aumento también de su mantenimiento, lo cual sucede porque al tener una buena planeación se analizan los requerimientos de espacio y necesidades específicas de mantenimiento de la infraestructura vial en la ciudad. A su vez, cuando aumentan las construcciones y los mantenimientos se presenta una mayor y adecuada infraestructura que se adecúa a los requerimientos de la demanda de transporte, además de esto al haber aumentos en la infraestructura vial se disminuyen los accidentes, pues una de sus causas es el estado de la malla vial [18]. Un aumento de los mantenimientos viales genera aumento en la congestión vial debido al proceso que se le hace a la vía, mientras se encuentra en mantenimiento el tramo está fuera de operación, reduciéndose la capacidad de la vía, es por ende que la velocidad promedio se disminuye.

La última relación que se puede encontrar con la planeación de la movilidad es la ilegalidad, un aumento en la planeación lleva a la disminución de la ilegalidad, a causa de que si se tiene una buena planeación se puede llegar a la totalidad de la población para que esta no se vea desamparada por el sistema de movilidad. Por su parte y como es lógico, el transporte ilegal hace que el parque automotor aumente debido a que dicho transporte rueda por las vías de la ciudad, a su vez el parque automotor tiene influencia sobre cuatro variables: contaminación, tasa de ocupación vial, infraestructura vial y congestión; en la primera de ella con el aumento del parque automotor se presenta aumento en las emisiones que se realizan al ambiente y por ende a la contaminación.

Cuando se incrementa el parque automotor aumenta la tasa de ocupación vial, puesto que la capacidad de las vías se mantiene y a su vez los vehículos aumentan, lo que provoca un aumento en la tasa de ocupación vial. Finalmente se puede concluir que un aumento de la tasa de ocupación vial genera un aumento en la congestión por que la cantidad de carros circulante aumenta, además el aumento de la congestión también se ve expresado por el aumento del parque automotor, debido a que las capacidades viales se utilizan en mayor porcentaje. Por último, al aumento del parque automotor se presenta una reducción en la infraestructura vial, ya que esta sufre mayor deterioro por el paso de mayor cantidad de vehículos.

Por su parte, existe la cultura ciudadana que influencia tres variables más: ilegalidad, parque automotor y accidentalidad. A un aumento de la cultura ciudadana se obtiene una disminución de las tasas de accidentalidad, pues estas se presentan en mayor medida por imprudencias de los conductores y peatones. Además de lo anterior, el aumento de la cultura ciudadana ocasiona una disminución en la ilegalidad ya que la gente usa los sistemas legales y permitidos 
para transportarse. Por último, el aumento en la cultura ciudadana disminuye el parque automotor circulante en tanto se tienen hechos culturales, como compartir el carro para que se transporten más personas en un solo vehículo.

El aumento en la edad del parque automotor origina mayor contaminación a causa de los procesos de quema de combustible que sufren los carros con años de antigüedad, además a mayor edad del parque automotor se produce una reducción en la calidad del transporte ocasionado por factores de percepción de las personas transportadas y a la vida útil de los automóviles de servicio de transporte público; a su vez, la calidad del transporte público es influenciada por la velocidad promedio, un aumento en la velocidad promedio genera aumento en la calidad del transporte público, puesto que la velocidad es el factor primordial en las necesidades de movilización de pasajeros.

En la construcción del diagrama causal mostrado en la figura 4, se tuvieron en cuenta los siguientes subsistemas:

- Compra de automóviles

- Parque automotor

- Motocicletas

- Taxis

- Transporte público colectivo

- Calidad del transporte

- Ordenamiento territorial

- Accidentalidad

- Estructura institucional

- Congestión vial

- Modelo empresarial

- Ilegalidad

- Tasa de ocupación vial

- Desintegración del sistema

- Sobreoferta

- Integración vial

De los anteriores diagramas se puede concluir que las relaciones entre variables no son lineales, por lo que la simulación con Dinámica de Sistemas se convierte en una herramienta adecuada para abordar esta problemática; aunado a lo anterior, el sistema presenta un gran nivel de complejidad a causa de la alta interacción entre variables. 


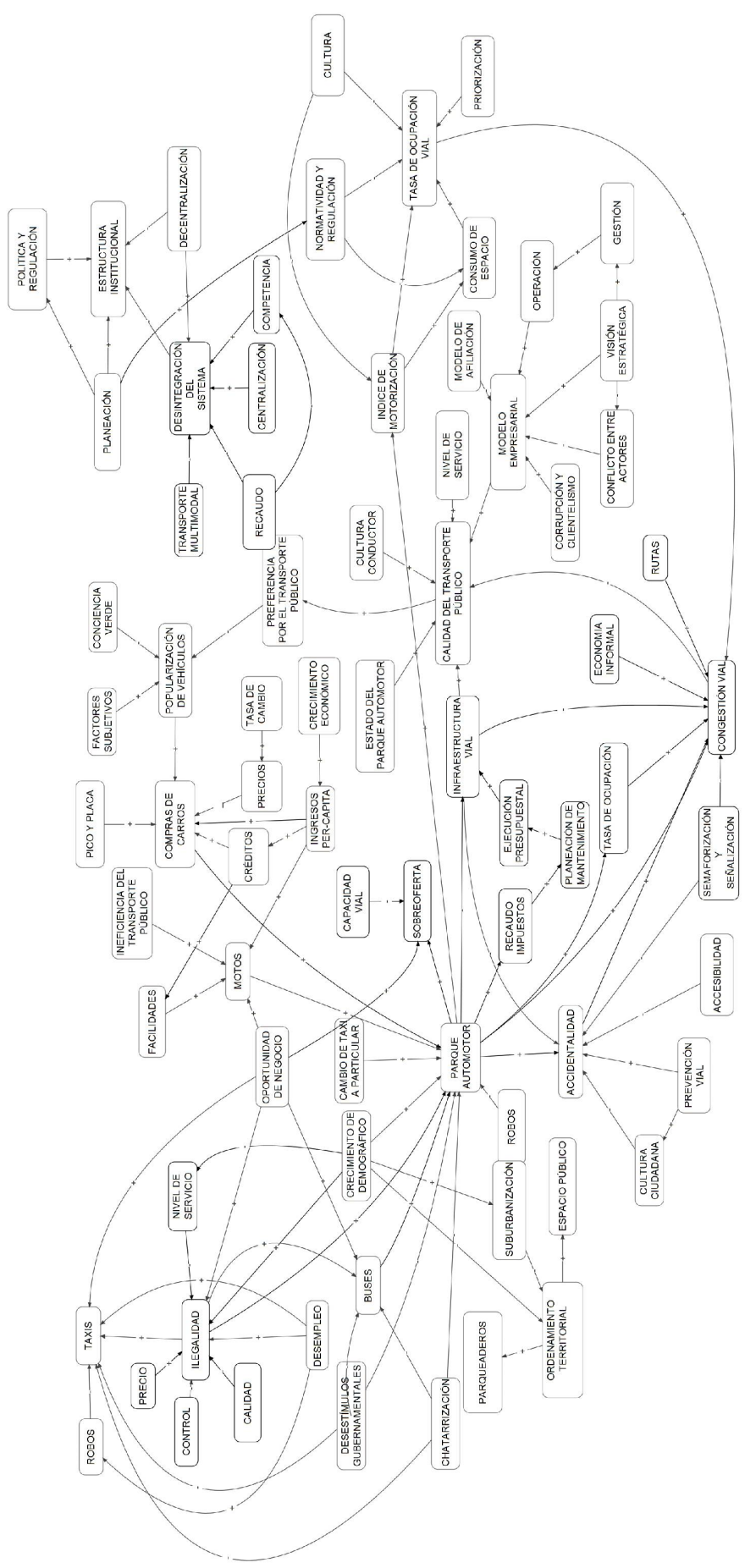

Figura 4. Diagrama específico. 


\subsection{Modelo de simulación}

Para el desarrollo del modelo de simulación se utilizaron trece sectores que representan la simulación dinámica del sistema de transporte de Bogotá D.C., el modelo de simulación se construyó con base en el software I-Think, el cual permite realizar las relaciones existentes entre las variables. Los sectores que se utilizaron para la simulación son:

- Porcentajes de ocupación por modo

- Indicadores de consumo por modo

- Velocidad promedio

- Congestión vial

- Velocidad promedio Transmilenio

- Accidentalidad

- Calidad Transmilenio

- Calidad transporte público colectivo

- Subsistema vial

- Parque automotor motocicletas

- Parque automotor privado

- Parque automotor transporte público individual

- Parque automotor transporte público colectivo

Los datos utilizados para la simulación provienen de la Secretaria de Movilidad, por tanto tienen un alto nivel de confianza para el análisis respectivo. En la figura 5 se muestra uno de los sectores de la simulación construida (para conocer el modelo completo se recomienda revisar la tesis de pregrado Evaluación de la problemática de transporte en Bogotá D.C., utilizando Dinámica de Sistemas, Universidad Distrital Francisco José de caldas); en este diagrama se ilustra el sector usando un diagrama de Forrester [19], representando así las variables: flujos, tasas y las interacciones entre las distintas variables.

Para el desarrollo del modelo del parque automotor del transporte público colectivo urbano se tuvieron en cuenta una serie de características que determinaron su estructura, las características tenidas en cuenta se mencionan a continuación:

- Los procesos de chatarrización y reinserción

- Edad promedio del parque automotor

- Ilegalidad

- Pico Placa, normal y ambiental 


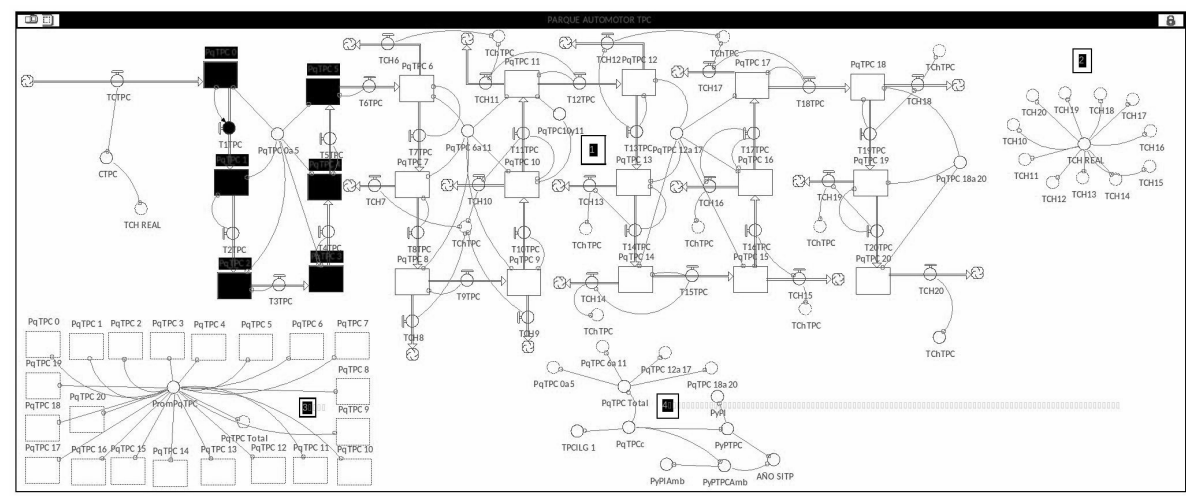

Figura 5. Diagrama de Forrester parque automotor del transporte público.

Sobre la base de lo anterior, el parque automotor se considera como un nivel que se drena por el número de buses que se chatarrizan y que aumenta con el número de buses que son reinsertados. Es importante mencionar que este gran nivel que contiene a todos los buses, busetas y colectivos se desagregó en varios niveles con el fin de conocer el número de vehículos por rango de edad, lo que implicó realizar un modelo de transición de años.

Los buses transitan de un nivel a otro, en otras palabras, incrementen un año por cada paso de la simulación, esta representación se realizó porque era necesario conocer la edad promedio de los vehículos y la cantidad de vehículos candidatos a ser chatarrizados (veinte años o mayores).

En la representación se establece cuatro secciones, la primera y más grande corresponde a los niveles del parque automotor; la segunda que encierra una serie de variables auxiliares en donde se calcula la tasa de chatarrización real; la tercera que se halla representada por todos los niveles y sirve para el cálculo de la edad promedio del parque automotor; y la cuarta en la que se calcula la tasa de vehículos circulantes. En la primera se relacionan cuatro tipos de elementos. Los niveles son la cantidad de vehículos por rango de edad y están identificados por PqTPC \#, donde \# señala la edad; las tasas de transición de un año a otro, representadas por T\#TPC, donde \# es el año en el cual transitan los vehículos.

A fin de realizar los análisis de la información suministrada se tuvieron en cuenta las relaciones entre las variables, para así tener en cuenta la dinámica del sistema de transporte junto con técnicas estadísticas, con el propósito de obtener relaciones entre variables.

\subsection{Análisis de políticas}

Una vez realizados los análisis correspondientes y el modelo de simulación de los puntos anteriores, se procede a evaluar sobre este modelo diferentes políticas que han sido señaladas por los expertos consultados como la solución a la problemática de transportes, las políticas evaluadas son las siguientes:

- Escenario tendencial: consiste en analizar las condiciones del sistema actual de transporte si no se realiza ningún cambio en el mismo. 
- Sistema integrado de transporte: representa la implementación total del sistema integrado de transporte sin metro.

- Sistema integrado de transporte con metro: consiste en la implementación del sistema integrado de transporte con metro.

- Malla vial: evalúa la recuperación de la malla vial y el crecimiento de esta.

- Escenario utópico: es la reunión de todas las soluciones anteriormente mencionadas.

Los supuestos utilizados en la simulación son los siguientes:

- La cantidad de vehículos circundantes por reglamentación distrital son 12300.

- En los escenarios del sistema integrado y sistema integrado con metro existe un cambio modal del $20 \%$ y $30 \%$ respectivamente, lo cual representa la cantidad de personas que dejan de utilizar transporte individual para usar el transporte colectivo.

- El escenario de malla vial contempla mejoras del 10\%, 20\%, 30\% y $40 \%$ para evaluar el impacto en la velocidad promedio, estas mejoras indican que las vías que se hallan en mal estado, pasan a ser buenas a una tasa diferente a la regular.

Para analizar los resultados de las políticas se tomó como medida de desempeño la velocidad promedio, ya que es la medida directamente relacionada con el tiempo de los viajes y desplazamientos en el sistema de transporte de la ciudad. En las figuras 6, 7, 8, 9 y 10 se muestran los resultados arrojados por el software con respecto al desempeño de la velocidad promedio con el paso del tiempo; junto a estas figuras, en las tablas IV, V, VI, VII y VIII se muestran los resultados arrojados por cada escenario, en las tablas se expone una simulación realizada hacia el año 2030 en donde se contrastan los resultados del indicador en el año 2010 versus el mismo indicador en el año 2030. Los indicadores que se contrastan son: velocidad promedio, parque automotor circulante, porcentaje de pasajeros transportados por modo de transporte y el aporte a congestión por modo de transporte. En el escenario de malla vial se presenta el resultado de la mejora de la malla vial junto con el resultado de la velocidad promedio en el año 2030 .

Tabla IV. Resultados escenario tendencial

\begin{tabular}{lc}
\hline Media & Valor \\
\hline Velocidad promedio 2010 & $20,70 \mathrm{Km} / \mathrm{h}$ \\
Velocidad promedio 2030 & $19,52 \mathrm{Km} / \mathrm{h}$ \\
Parque automotor circulante 2010 & 722,952 \\
Parque automotor circulante 2030 & 890,686 \\
\% transportado por motos 2010 & 5,43 \\
\% transportado por motos 2030 & 7,92 \\
\% transportado por carros 2010 & 30,16 \\
\% transportado por carros 2030 & 20,87
\end{tabular}




\begin{tabular}{lc} 
\% transportado por transmilenio 2010 & 14,34 \\
$\%$ transportado por transmilenio 2030 & 28,2 \\
$\%$ transportado por buses 2010 & 46,19 \\
$\%$ transportado por buses 2030 & 41,19 \\
$\%$ transportado por taxis 2010 & 3,87 \\
$\%$ transportado por taxis 2030 & 1,84 \\
$\%$ aporte a congestión carros 2030 & 79,73 \\
$\%$ aporte a congestión motos 2030 & 14,36 \\
$\%$ aporte a congestión buses 2030 & 2,58 \\
$\%$ aporte a congestión taxis 2030 & 3,34 \\
\hline
\end{tabular}

Tabla V. Resultados escenario sistema integrado sin metro

\begin{tabular}{|c|c|}
\hline Media & Valor \\
\hline Velocidad promedio 2010 & $20,82 \mathrm{Km} / \mathrm{h}$ \\
\hline Velocidad promedio 2030 & $17,95 \mathrm{Km} / \mathrm{h}$ \\
\hline Parque automotor circulante 2010 & 722,952 \\
\hline Parque automotor circulante 2030 & $1,047,866$ \\
\hline$\%$ transportado por motos 2010 & 5,77 \\
\hline$\%$ transportado por motos 2030 & 7,59 \\
\hline$\%$ transportado por carros 2010 & 32,04 \\
\hline$\%$ transportado por carros 2030 & 23,53 \\
\hline$\%$ transportado por transmilenio 2010 & 15,23 \\
\hline$\%$ transportado por transmilenio 2030 & 27,02 \\
\hline$\%$ transportado por buses 2010 & 42,84 \\
\hline$\%$ transportado por buses 2030 & 40,11 \\
\hline$\%$ transportado por taxis 2010 & 4,11 \\
\hline$\%$ transportado por taxis 2030 & 1,76 \\
\hline$\%$ aporte a congestión carros 2030 & 82,23 \\
\hline$\%$ aporte a congestión motos 2030 & 12,59 \\
\hline$\%$ aporte a congestión buses 2030 & 2,26 \\
\hline$\%$ aporte a congestión taxis 2030 & 2,93 \\
\hline
\end{tabular}


1: VELOCIDAD PROMEDIO

$1:$

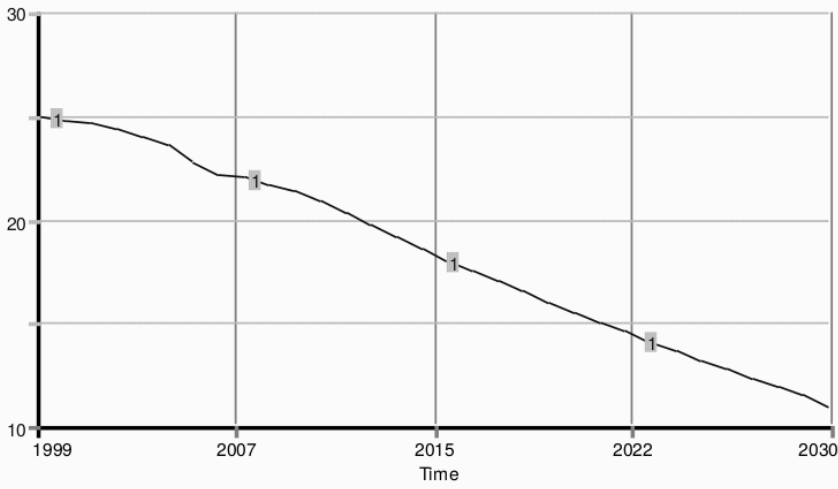

?

Velocidad promedio

Figura 6. Velocidad promedio escenario tendencial.

1: VELOCIDAD PROMEDIO

1

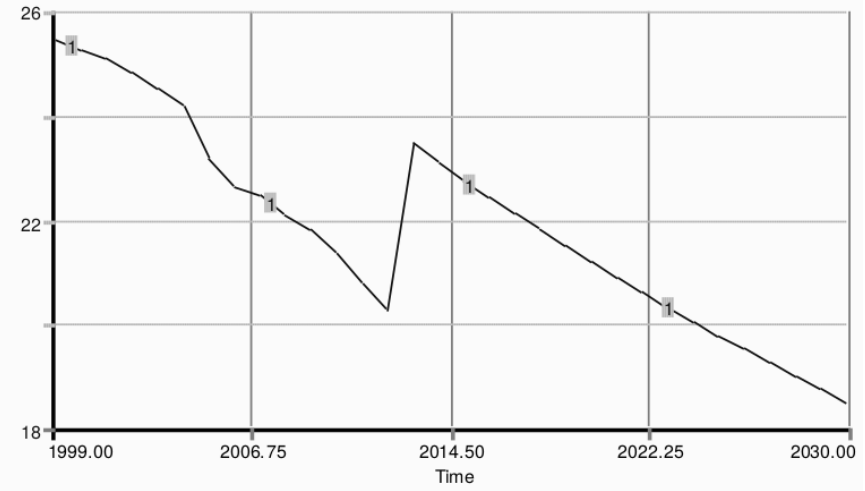

?

Velocidad Promedio

Figura 7. Velocidad promedio sistema integrado de transporte sin metro.

1: VELOCIDAD PROMEDIO

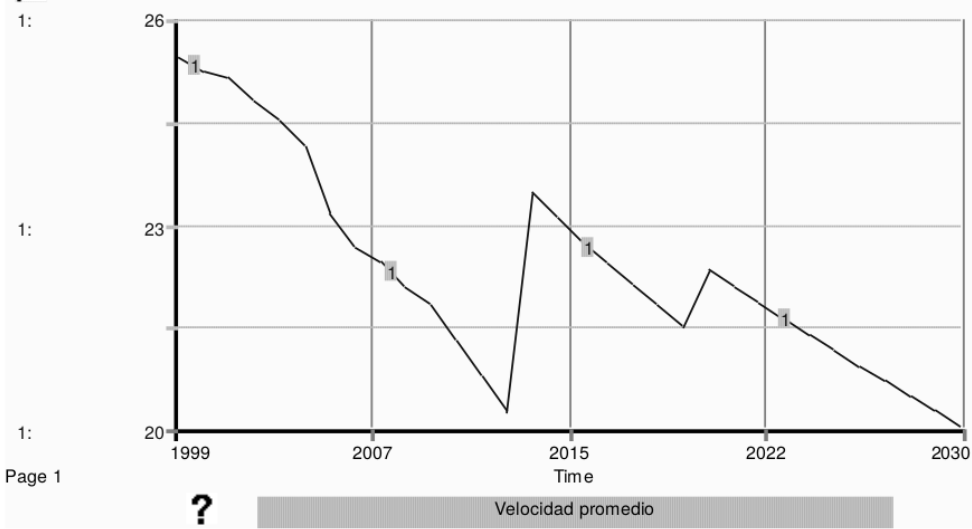

Figura 8. Velocidad promedio sistema integrado de transporte con metro. 
Tabla VI. Resultados escenario sistema integrado con metro

\begin{tabular}{|c|c|}
\hline Media & Valor \\
\hline Velocidad promedio 2010 & $20,77 \mathrm{Km} / \mathrm{h}$ \\
\hline Velocidad promedio 2030 & $19,52 \mathrm{Km} / \mathrm{h}$ \\
\hline Parque automotor circulante 2010 & 722,952 \\
\hline Parque automotor circulante 2030 & 890,686 \\
\hline$\%$ transportado por motos 2010 & 5,43 \\
\hline$\%$ transportado por motos 2030 & 7,92 \\
\hline$\%$ transportado por carros 2010 & 30,16 \\
\hline$\%$ transportado por carros 2030 & 20,87 \\
\hline$\%$ transportado por transmilenio 2010 & 14,34 \\
\hline$\%$ transportado por transmilenio 2030 & 28,2 \\
\hline$\%$ transportado por buses 2010 & 46,19 \\
\hline$\%$ transportado por buses 2030 & 41,19 \\
\hline$\%$ transportado por taxis 2010 & 3,87 \\
\hline$\%$ transportado por taxis 2030 & 1,84 \\
\hline$\%$ aporte a congestión carros 2030 & 79,73 \\
\hline$\%$ aporte a congestión motos 2030 & 14,36 \\
\hline$\%$ aporte a congestión buses 2030 & 2,58 \\
\hline$\%$ aporte a congestión taxis 2030 & 3,34 \\
\hline
\end{tabular}

VELOCIDAD PROMEDIO: $1-2-3-4$.

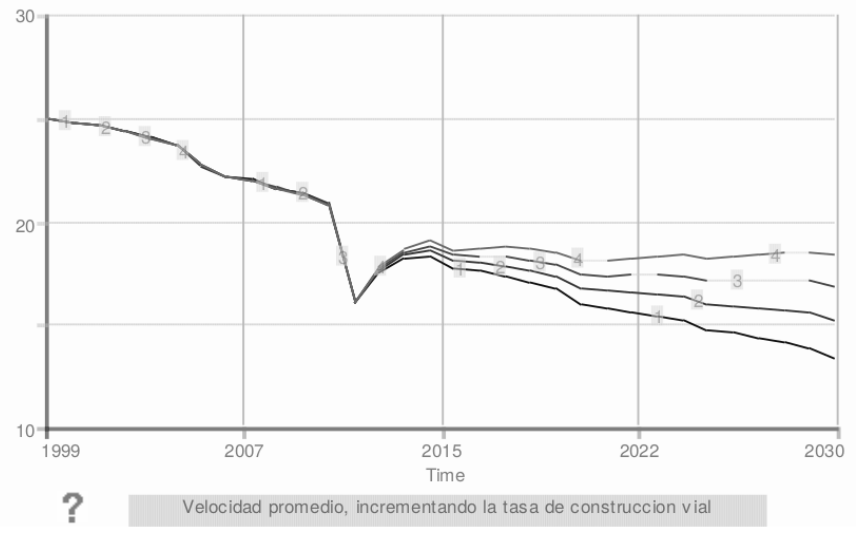

Figura 9. Velocidad promedio mejoramiento de la malla vial. 
Tabla VII. Resultados escenario mejoramiento de la malla vial

\begin{tabular}{lc}
\hline Media & Valor \\
\hline Velocidad promedio 2030 incremento $10 \%$ malla vial & $13 \mathrm{~km} / \mathrm{h}$ \\
Velocidad promedio 2030 incremento $20 \%$ malla vial & $15 \mathrm{~km} / \mathrm{h}$ \\
Velocidad promedio 2030 incremento $30 \%$ malla vial & $17 \mathrm{~km} / \mathrm{h}$ \\
Velocidad promedio 2030 incremento $40 \%$ malla vial & $18 \mathrm{~km} / \mathrm{h}$ \\
Malla vial buena 2010 & $5237 \mathrm{~km}$ \\
Malla vial buena 2030 & $18463 \mathrm{~km}$ \\
Malla vial regular 2010 & $3364 \mathrm{~km}$ \\
Malla vial regular 2030 & $2281 \mathrm{~km}$ \\
Malla vial mala 2010 & $6322 \mathrm{~km}$ \\
Malla vial mala 2030 & $1105 \mathrm{~km}$ \\
\hline
\end{tabular}

1: VELOCIDAD PROMEDIO

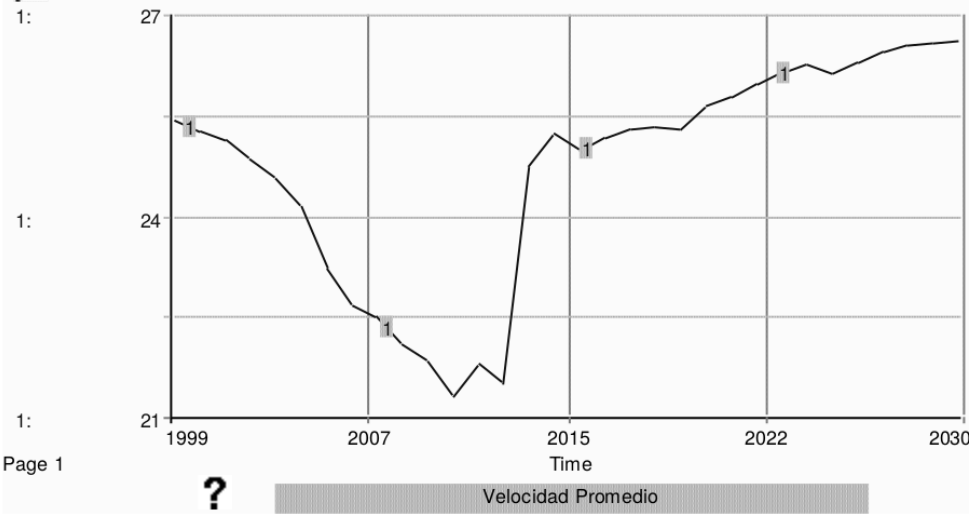

Figura 10. Velocidad promedio escenario utópico.

Tabla VIII. Resultados escenario utópico

\begin{tabular}{lc}
\hline Media & Valor \\
\hline Velocidad promedio 2030 incremento 10\% malla vial & $20,78 \mathrm{~km} / \mathrm{h}$ \\
Velocidad promedio 2030 incremento $20 \%$ malla vial & $26,11 \mathrm{~km} / \mathrm{h}$ \\
Velocidad promedio 2030 incremento $30 \%$ malla vial & 722,952 \\
Velocidad promedio 2030 incremento $40 \%$ malla vial & 556,678 \\
Malla vial buena 2010 & 5,5 \\
Malla vial buena 2030 & 6,51 \\
Malla vial regular 2010 & 30,51 \\
Malla vial regular 2030 & 28,62
\end{tabular}


Malla vial mala 2010

14,5

Malla vial mala 2030

32,2

$\%$ transportado por buses 2010

45,58

$\%$ transportado por buses 2030

40,16

$\%$ transportado por taxis 2010

$\%$ transportado por taxis 2030

$\%$ aporte a congestión carros 2030

71,08

$\%$ aporte a congestión motos 2030

20,48

$\%$ aporte a congestión buses 2030

Teniendo en cuenta los resultados ilustrados en las figuras 6 a 9 se puede concluir que ninguna de las medidas implementadas tiene una influencia sobre la velocidad promedio continua en el tiempo, mostrando así que ninguna medida ejecutada por separado soluciona el problema de manera definitiva.

En la figura 6 se observa cómo disminuye la velocidad con el paso del tiempo ya que no existe ningún tipo de intervención para la regulación del uso del transporte público y para el cambio modal hacia el sistema integrado, se puede observar en los resultados mostrados en la tabla IV que el mayor aporte a la congestión se produce por el uso del automóvil al tener un valor cercano al 90

En las figuras 7 y 8 se observa la implementación del sistema integrado de transporte público sin y con metro respectivamente, lo que se puede evidenciar es que se presentan unos incrementos o mejoras sustanciales pero no perduran en el tiempo, lo cual se debe a la implementación de las medidas que tienen un impacto en la percepción y el uso modal, pero con el paso del tiempo no es suficiente y el sistema tiende a bajar nuevamente; como se puede observar en las tablas V y VI, existe una mejora en el indicador de velocidad promedio en comparación con el escenario anterior, también hay un cambio del modo vial, es decir, el porcentaje de pasajeros transportados en automóvil es menor, sin embargo este cambio no es significativo para el sistema y el porcentaje de personas que usan vehículo privado es mayor.

En la figura 9 se puede ver el mejoramiento en la velocidad promedio dependiendo de las mejoras en la malla vial, si estas aumentan en un $10 \%, 20 \%, 30 \%$ y $40 \%$ respectivamente; pero al igual que las medidas anteriores estas mejoras no perduran en el tiempo, de la misma forma que se muestra en la tabla VII ya que se observa cómo se dan las mejoras de la maya vial en el tiempo pero las velocidades no perduran.

De manera contraria en la figura 10, se muestra cómo en el escenario utópico la velocidad promedio mejora con el paso del tiempo, lo que demuestra que para solucionar una problemática social es necesario tener en cuenta todos los factores y no realizar un análisis separado de los mismos; así, se puede ver en la tabla VIII que el indicador de velocidad promedio al final de la simulación tiene una tendencia de crecimiento y el porcentaje de aporte 
a la congestión por parte de los carros es cercano al $70 \%$, que es el mayor valor de todas los escenarios que se tuvieron en cuenta.

\section{Conclusiones}

Con el desarrollo de la presente investigación se puede mostrar la aplicación de un modelo dinámico para la estudio y evaluación de un fenómeno social, para este caso específico se abordó la temática de transporte en la ciudad de Bogotá D.C., con la obtención del modelo se pueden analizar las condiciones del sistema de transporte, además este modelo permite tener medidas cuantitativas, las cuales no son usadas frecuentemente en los sistemas sociales. El uso de este tipo de metodología para la caracterización de sistemas involucra una serie de dificultades inmersas en el comportamiento de los sistemas sociales, que se reflejan en el número de variables, el número de relaciones y el tipo de relaciones que crean. Con respecto a la metodología utilizada se puede concluir lo siguiente:

- En el proceso de recolección de información de expertos se pueden encontrar contradicciones respecto a temáticas específicas, y al no contar con modelos matemáticos que ayuden a su interpretación, no se puede dar acierto a alguna de dichas opiniones, es por tanto que con este tipo de investigaciones es viable proporcionar medidas numéricas que ayuden a resolver dichas discrepancias.

- Al realizar el diagrama de Pareto se encontró que no se podía demostrar el 80-20 planteado por este principio, por lo que se concluye que todas las características planteadas que describen la problemática tienen igual grado de impacto sobre el sistema de movilidad.

- Se puede concluir que es imposible tener una visión lineal de las causas de la problemática referente a esta investigación, debido a que al realizar el diagrama de causa-efecto se encontraron algunas causas principales, que a su vez eran causas que explicaban otras causas principales.

- El diagrama causal permite la búsqueda de las relaciones de las diferentes variables. Es importante señalar que la complejidad de un sistema no se mide exclusivamente por el número de variables, sino por el número de interrelaciones que tiene dicho sistema, razón por la cual es indispensable utilizar este acercamiento con fin de entender el sistema.

La implementación de un modelo matemático de simulación continua permite diagnosticar las situaciones futuras y se constituye en una herramienta que sirve para la toma de decisiones, además en este modelo se simularon seis diferentes escenarios para verificar el impacto en las políticas sobre el sistema; en estos escenarios se muestra la evolución de las condiciones del transporte en Bogotá D.C. a través del tiempo. 
Con el análisis de escenarios se puede concluir lo siguiente:

- En todos los escenarios se muestra una mejora en el indicador principal (velocidad promedio), esto demuestra que el problema del transporte público en Bogotá D.C. es un problema de capacidades, el cual debe ser analizado desde la capacidad requerida, las personas transportadas y la capacidad disponible.

- La solución que se implante al sistema de movilidad tiene que contemplarse dentro de los campos de capacidad requerida y capacidad disponible.

- Por último se puede señalar que el escenario utópico, que reúne todas las políticas, es el único que trae mejoras al sistema perdurables en el tiempo, es por tanto que no se pueden dar veredictos sobre las demás políticas puesto que estas solo traen beneficios en periodos cortos de tiempo, por lo que se hace indispensable establecer estrategias que involucren a todo el sistema sin centrarse demasiado en cosas específicas.

Por último cabe resaltar que existen factores socioculturales, económicos y políticos que deben resolverse con el fin de promover medidas que impacten positivamente en el transporte, y algo aún más importante, que los beneficios que traigan se mantengan en el tiempo.

Como trabajos de investigación futuros existen escenarios que no se tuvieron en cuenta en el modelo de simulación como el uso de medios de transporte alternativos (bicicletas y otros medios alternos), ampliaciones viales, comportamientos de los peatones en el uso de medios de transporte y el creciente fenómeno del aumento de las motocicletas. También se pudieron observar comportamientos de los resultados de la malla vial que podrían ser simulados mediante cadenas de Markov.

\section{Referencias}

[1] Programa de las Naciones Unidas para el Desarrollo, informe sobre Desarrollo Humano, Superando barreras: Movilidad y desarrollo humano, 2009, disponible en http://hdr.undp.org

[2] Germán Lleras, "Transmilenio y el transporte colectivo tradicional, una relación incierta”. Revista de Ingeniería, 21, junio 2005, pp. 84-93.

[3] Jay Forrester, World Dynamics. Wright-Allen Press, Massachusetts, Estados Unidos, 1971, 15.

[4] Germán Méndez y Lindsay Álvarez, Diseño de prototipo diagnóstico para la pequeña y mediana empresa, pyme. Nomos, Bogotá D.C., Colombia, 2004, pp. 21-32.

[5] Indra Khanna, N. Singh y Prem Vatt, "System dynamics in urban transportation planning and policy analysis". Proceedings Keystone CO USA, 1986, pp. 453-462.

[6] Khaled Abbas, "The use of system dynamics in modelling transportation systems with respect to new cities in Egypt". 8th International conference of the Systems Dynamics Society, Chesnut Hill, Masachussets, Estados Unidos, 1990, pp. 16-30

[7] Jifeng Wang, Huapu Lu y Hu Peng, "System Dynamics model of urban transportation system and its applications”. Journal of Transportation Systems engineering and information technology, 8, 3, junio 2008, pp. 83-89.

[8] Hossein Haghshenas, Manouchehr Vaziri y Ashkan Gholamialam, "Evaluation of sustainable policy in urban transportation using system dynamics and world cities data: A case study in Isfahan". Cities, in Press, noviembre 2014. 
[9] J.-H. Lew, L. F. Hivin y D.N. Mavris, "A multi-paradigm approach to system dynamics modeling of intercity transportation". Transportation Research Part E: Logistics and Transportation Review, 71, noviembre 2014, pp. 188-202.

[10] Yang Yang, Peitong Zhang y Shaoquan Ni, "Assessment of the impacts of urban rail transit on metropolitan regions using systems dynamics model". Transportation Research Proceedings, 4, mayo 2014, pp. 521-534.

[11] Edgar Duarte, "El transporte público colectivo en Bogotá D.C.: una mirada desde la Dinámica de Sistemas". Ingeniería, 16, 2, noviembre 2011, pp. 18-34.

[12] J. Orozco, F. Arenas, "Aproximación al desarrollo de un sistema de transporte masivo a través de la Dinámica de Sistemas". Revista S\&T, 11, 24, mayo 2013, pp. 91-106.

[13] Martin Schaffernicht, Aplicación del análisis de sistemas a las ciudades y al transporte público urbano, innovación ambiental de servicios urbanos y de infraestructura: hacia una economía baja en carbono. Comisión económica para América Latina y el Caribe, Santiago de Chile, Chile, 2012, pp. 9-102.

[14] Diagrama de Pareto, Dirección nacional de servicios académicos virtuales, Universidad Nacional de Colombia sede Manizales, 2010, disponible en http://www.virtual.unal.edu.co

[15] G. Levaggi, La teoría general de los sistemas. 2, Ugerman, Tucumán México, 2007, pp. 20-171.

[16] J. Arango, "Calidad de los combustibles en Colombia". Revista de Ingeniería, 29, mayo 2009, pp. 100-108.

[17] Ian Thomson, Alberto Bull, "La congestión del tránsito urbano: causas y consecuencias económicas y sociales". Revista de la CEPAL, 16, abril 2002, 109-121.

[18] Observatorio de Movilidad, Boletín de accidentalidad vial, Boletín No 2, 2004, disponible en http://www.movilidadbogota.gov.co/hiwebx_archivos/audio_y_video/boletin \%20de \%20cifras \%2011-072012.pdf

[19] Jay Forrester, Industrial dynamics. Wright-Allen Press, Massachusetts Estados Unidos, 1961, pp. 40-52.

[20] Javier Aracil, Dinámica de sistemas. Isdefe, Madrid España, 1995, pp. 21-49.

\section{Carlos Franco Franco}

Nació en Bogotá, Colombia. Es Ingeniero Industrial de la Universidad Distrital Francisco José de Caldas, Bogotá, Colombia. Obtuvo su título de Maestría en Ingeniería Industrial en la Universidad de los Andes, Bogotá, Colombia. Actualmente se desempeña como profesor en el área de investigación de operaciones y logística en la Universidad Distrital Católica de Colombia, Bogotá, Colombia, y pertenece como investigador al grupo Grupo de Investigación en Gestión Industrial (GEGI).

e-mail: cafranco@ucatolica.edu.co

\section{Germán Andrés Méndez Giraldo}

Nació en Bogotá, Colombia. Es Ingeniero Industrial de la Universidad Distrital Francisco José de Caldas, de Bogotá, Colombia. Obtuvo su título de Maestría en Ingeniería Industrial en la Universidad de los Andes de Bogotá, Colombia, 1998. Obtuvo su Doctorado en Ciencias Técnicas en la Universidad de las Villas, Santa Clara, Cuba, 1999. Se desempeñó como Jefe de Producción en industrias nacionales y multinacionales, Gerente y Coordinador de proyectos. Consultor y asesor empresarial. Es profesor titular de la Facultad de Ingeniería de la Universidad Distrital desde 1995 y se ha desempeñado en diversos cargos dentro de la Institución como coordinador de la Especialización en Ingeniería de Producción, de la Maestría en Ingeniería Industrial, fue Jefe de la Ofi cina de Relaciones Interinstitucionales y Vicerrector Administrativo y fi nanciero de la Universidad Distrital. Actualmente se desempeña como profesor en el área de investigación de operaciones en la Universidad Distrital Francisco José de Caldas, Bogotá, Colombia, y pertenece como investigador y director del grupo Sistemas Expertos y Simulación donde realiza estudios sobre sistemas expertos, dinámica de sistemas y optimización.

e-mail: gmendez@udistrital.edu.co

\section{John Espitia}

Es Ingeniero Industrial de la Universidad Distrital Francisco José de Caldas, Bogotá, Colombia. Obtuvo su título de Maestría en Ingeniería Industrial en la Universidad de los Andes, Bogotá, Colombia. Actualmente se desempeña como consultor independiente.

e-mail: jaerjr@gmail.com 